\title{
Use of Machine Learning by Non-Expert DHH People: Technological Understanding and Sound Perception
}

\author{
Yuri Nakao* \\ nakao.yuri@fujitsu.com \\ Fujitsu Limited. \\ Kawasaki, Japan
}

\author{
Yusuke Sugano \\ sugano@iis.u-tokyo.ac.jp \\ The University of Tokyo \\ Tokyo, Japan
}

\begin{abstract}
Recent advances in machine learning demonstrated its potential in accessibility applications. However, recognition models and their application scenarios are often defined by machine learning (ML) experts and cannot fully capture users' diverse demands with disabilities. In order to open up the full potential of ML for accessibility applications, we have to bridge the gap for non-expert people doubly caused by the technological understanding and their disabilities. In this work, we investigate how non-expert deaf and hard-of-hearing $(\mathrm{DHH})$ people understand ML technologies and design ML-based sound recognition systems. We conduct a workshop study consisting of an ML lecture and an interactive learning session using a sound recognition system. Through observations during the workshop and semi-structured interviews, we clarify that non-expert DHH people start to overcome the knowledge gap. They could obtain a more detailed understanding of ML technology and how to use sounds to train ML models.
\end{abstract}

\section{CCS CONCEPTS}

- Social and professional topics $\rightarrow$ People with disabilities; • Computing methodologies $\rightarrow$ Machine learning.

\section{KEYWORDS}

Accessibility, Machine Learning, Sound Recognition

\section{ACM Reference Format:}

Yuri Nakao and Yusuke Sugano. 2020. Use of Machine Learning by NonExpert DHH People: Technological Understanding and Sound Perception. In Proceedings of the 11th Nordic Conference on Human-Computer Interaction: Shaping Experiences, Shaping Society (NordiCHI '20), October 25-29, 2020, Tallinn, Estonia. ACM, New York, NY, USA, 12 pages. https://doi.org/10. $1145 / 3419249.3420157$

\section{INTRODUCTION}

Recent advances in statistical machine learning (ML) have shown great potential for improving assistive technologies with better accuracy and diverse ranges of recognition tasks [31, 32]. Image

\footnotetext{
*Also with Fujitsu Laboratories Ltd..

Permission to make digital or hard copies of all or part of this work for personal or classroom use is granted without fee provided that copies are not made or distributed for profit or commercial advantage and that copies bear this notice and the full citation on the first page. Copyrights for components of this work owned by others than the author(s) must be honored. Abstracting with credit is permitted. To copy otherwise, or republish, to post on servers or to redistribute to lists, requires prior specific permission and/or a fee. Request permissions from permissions@acm.org.

NordiCHI '20, October 25-29, 2020, Tallinn, Estonia

(c) 2020 Copyright held by the owner/author(s). Publication rights licensed to ACM ACM ISBN 978-1-4503-7579-5/20/10 . \$ \$15.00

https://doi.org/10.1145/3419249.3420157
}

and sound recognition technologies, for example, can help visuallyimpaired and deaf and hard-of-hearing (DHH) people by providing additional sensory modalities. Hearing loss is known as one of the most common disability, and some existing studies have investigated the potential of ML-based assistive technology to support DHH people in some specific scenarios, such as detecting sounds of ringing doorbells and crying people $[4,33]$. However, compared to the case of visual recognition, it is more difficult to support $\mathrm{DHH}$ people with ML because of the difficulty of representing/visualizing audio data.

Despite the advances of ML technology, the knowledge required to design ML applications is still not fully democratized and the potential of ML is not entirely opened up to non-experts. It has been considered important for assistive technologies to be designed by people with disabilities themselves because the technologies directly influence their daily lives, and people with disabilities have to decide on their own what and how to solve their problems with technologies [20]. Almost no existing researches, however, investigated how to let people with disabilities make full use of the ML. Even when people with disabilities participate in the process of scenario settings, the experts took the leads, and non-experts with disabilities did not design their solution for themselves [4]. Ideally, there should be a situation where people with disabilities can freely investigate the potential solution space of ML to design their own solutions.

On the other hand, the key difficulty for non-expert DHH people to design ML-based assistive technologies is the knowledge gap doubly caused by the technological understanding and their disabilities. Several prior studies have already pointed out that the technological knowledge gap is one of the key challenges to democratize ML technologies in general. Non-expert people often fail to have appropriate mental models for ML-based tools [23, 36], or to cover sufficient factors when building or evaluating ML models [39]. For DHH people, the knowledge gap becomes even more critical because of their disabilities. For most of them, there is a difference between the actual environmental sound and the sound they imagine or they perceive. Therefore, they have difficulties in expressing the application scenarios based on the actual sound, and also imagining the training sound data required to perform the ML tasks.

The goal of this work is to investigate how the perception of technology and sound change through interactions with ML technologies. As a step toward the development of ML-based assistive technologies for $\mathrm{DHH}$ people where they can design their own solution for their daily problems, we analyze a workshop involving an interactive sound recognition system (Fig. 1). We prepare a system with a graphical user interface (GUI) that allows users to 

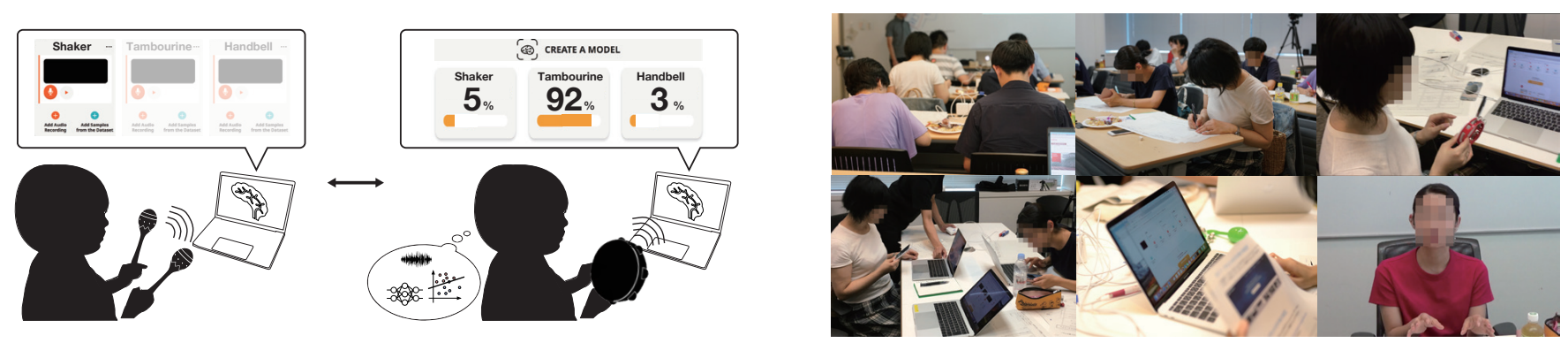

Figure 1: This study investigates how non-expert deaf and hard-of-hearing (DHH) people understand machine learning technology in the situation where there are not any definition of use-case scenario and recognition models.

train their own sound recognition models, and hold a workshop in which DHH participants use the interactive system after a lecture on ML. This allows us to investigate interactions between participants and ML systems while non-expert participants can explore the possibility of sound recognition models freely without strong guidance from experts nor pre-defined application scenarios. In this way, unlike prior workshop studies on non-ML assistive technologies $[13,27,40]$, we explore the scenario where non-expert users can define scenarios and tasks for themselves. We evaluate and analyze the effect of various aspects of the workshop process based on observations during the workshop, worksheet descriptions for the participants, and semi-structured interviews.

Our contributions are summarized as follows. First, to the best of our knowledge, we provide the first in-depth analysis of a workshop where $\mathrm{DHH}$ participants freely interact with an interactive sound recognition system and express ideas on ML tasks without expert guidance nor fixed use-case scenarios. Second, based on the analyses, we clarify how non-expert DHH people's understanding of ML changes along with the proceeding of the workshop, and how the workshop process has effects on the changes of their understandings of ML and the sounds. Finally, we highlight some possibilities for and challenges in, designing ML applications with DHH users involved and creating interactive ML systems to solve diverse daily-life problems.

\section{BACKGROUND AND RELATED WORK}

\subsection{Machine Learning in Assistive Technology}

There have been many efforts to extend the possibilities of assistive technology with ML technologies $[8,12,15]$. Unlike previous approaches to support sensory disabilities without ML including tactile devices, sensor networks [7, 34, 37], ML-based sensors can convey more complex information to users, and can potentially handle diverse demands from users. For example, ML-based speech recognition can be used to support DHH people [26, 38], and another prior study used ML to recognize environmental sound around DHH users [33]. However, despite the advances in ML-based sound recognition technologies $[6,14]$, it is still difficult to train a single recognition model to meet all requirements from diverse users. Due to the lack of the experts of ML, there are difficulties in having the experts design a custom-made ML solution for each user. The engineers and researchers usually do not share the same disability as the actual users, and do not fully understand the user demands.
To overcome the difficulties in grasping practical requirements from people with disabilities, a few prior studies designed ML-based application systems so that they can be personalized or adapted to the user requirements $[18,19,21]$. Kacorri et al. conducted structural research on an image recognition system for visually impaired people [19]. To overcome the issue of the limited amount of training data in the field of accessibility [18], they developed a framework for people with visual impairment to train their personalized classifiers. They let users define target recognition categories, and allowed them to train their own recognition models using their own training data [19]. However, though personalization allows users to change the target category under a pre-defined scenario, usually cannot fundamentally change the scenario itself (e.g., from personal object recognition to face identification from wearable cameras). To open up the full potential, the application scenario of ML-based recognition systems should be designed by users with disabilities, not by ML experts without disabilities.

Contrary to these prior work, in this study, we analyze a workshop where DHH participants can interact with a sound recognition system and freely explore ideas on ML applications to support their sensory disabilities. Our workshop process and the interactive system are designed to be as abstract as possible to represent any kinds of classification tasks so as not to restrict the application scenarios of sound recognition. By analyzing the expressed tasks and user behavior, we examine how users accept ML technology from the perspective of what kind of characteristics of users and technology have effects on the understandings of the technology.

\subsection{Interactive ML and Participatory Design}

To open up the opportunities of designing the ML technology for non-expert users, interactive ML has been actively studied in the HCI community [2,9]. The basic idea of interactive ML is to get users involved in an incremental training process by letting them inspect intermediate results and adjust training samples. For this purpose, some prior works have focused on human factors by studying how users interact with such systems [3, 10, 25, 28, 35]. In addition to the researches focusing on the users without disabilities, there have been a few prior studies applying interactive ML to support people with disabilities, such as people with learning and physical disabilities [21], for the purposes of making the systems personalized or meet the diverse needs of users. However, to the best of our knowledge, there have been no studies on interactive ML tools for DHH people. 
As an alternative way to enable end-users and researchers to develop technology interactively, several existing studies have applied the participatory design approach to assistive technology $[13,27,40]$. In the case of DHH users, auto-captioning systems $[22,30]$ and mock-up sound recognition systems [16] have been designed through participatory processes. While the prior studies above have not dealt with ML implementation, Bragg et al.'s work [4] focused on the development of interactive systems with ML to recognize specific sound categories, and conducted a Wizard-of-Oz-style experiment. While their study provided valuable insights into the daily-life needs of people with disabilities, strictly speaking, their use-case scenarios were still defined by the experts and restricted to specific applications. They investigated user demands through a selective questionnaire to design the use case, and this could potentially lead the task extraction to the answers set by the researchers in advance.

In this paper, we conduct a workshop study with the existence of an interactive sound recognition system as a tool to help $\mathrm{DHH}$ people understand ML. The system is designed for the purpose of avoiding restricting sound recognition to any specific application scenarios. Through our analyses of the change in the application ideas expressed by participants, we clarify the effects of using such an interactive system when DHH people understand ML, and we also investigate the design implications for interactive ML systems to support such users.

\section{WORKSHOP DESIGN}

The purpose of our workshop is to investigate how the non-expert DHH people's perception of ML technology and sounds changes through interactions with ML technologies. With this in our mind, we designed the workshop to let the participants learn the basic concepts of ML in both practical and theoretical senses, and to allow them to freely explore application ideas based on their daily-life demands. Our workshop consists of three main activities, as shown in Fig. 2: a lecture, interactive learning, and idea description. The lecture delivered by an ML expert is meant to convey theoretical knowledge of ML, while the interactive learning system is meant to complement the lecture by providing a practical experience of the statistical nature of ML systems. By asking the participants to write their ideas for sound recognition applications at multiple stages, we examine how the expressed tasks change as the experience of ML increases.

\subsection{Procedure}

As illustrated in Fig. 2, our workshop consists of six phases. In the first phase, the participants are instructed to fill out Worksheet A, which instructs them to 'describe as many concrete situations in your daily life as possible where you want to hear a specific sound or you have trouble due to the lack of a sound.' The worksheet is intended to let participants have clues to come up with the AI tasks without considering details of the ML technology, based on their daily demands. The worksheets are then shared among the participants to facilitate discussions between them and to let them know diverse requirements related to sound. In the second phase, we ask the participants to fill out Worksheet B, which asks participants to "choose three from the situations you described before, and describe concrete recognition tasks, i.e., what AI needs to recognize to solve your issue.' Participants are also allowed to describe more than three recognition tasks using multiple worksheets. In this phase, we intentionally use the phrase "AI" instead of "machine learning", so that the worksheets would reflect the participants' prior knowledge and expectations about AI.

The third phase is a short lecture by an ML expert, together with a demonstration of the interactive learning system. This is necessary because it has been pointed out that a deep mental model of complex systems can be formed only through a technical lecture, not through hands-on experience [24]. In the fourth phase, we ask the participants to fill in a Worksheet $\mathbf{C}$, which is similar to Worksheet B. The instruction on Worksheet $\mathbf{C}$ is: 'Based on what you learned from the lecture, please describe more concrete thoughts on how to train and realize the AIs and recognition tasks you described before using machine learning technologies. You can also write down any new recognition task ideas that you have come up with through the lecture.' The purpose of this worksheet is to examine the change of the expressed tasks due to the machine learning lecture.

In the fifth phase, the participants are asked to use the interactive system to train their own sound recognition systems. They are first asked to use Worksheet $\mathbf{D}$ to describe the recognition systems that they want to implement in detail, which do not have to correspond to the ideas described in the previous phases. We provide a laptop installed with the interactive system to each participant, and split the participants into small groups assigned different rooms respectively to avoid noise from other participants. Finally, in the sixth phase, the participants are asked to fill in Worksheet E, which is similar to Worksheet C. Worksheet $\mathbf{E}$ asks the participants that: 'Based on your experience with the sound recognition system, please describe if you come up with a better approach for training the AI and recognition task you described earlier. You can also write down any new recognition task ideas that you have come up with through your work.' This final worksheet is prepared to examine the change of the task expression after using the system.

\subsection{Interactive Learning System}

Figure 3 shows the GUI and flow of our interactive learning system. The GUI allows users to define sound categories, or classes, that they want to recognize and to add training samples to each class. They can train sound classifiers using their own training data, and validate the classification performance via live audio input. We develop the system via a prototyping process by conducting preliminary studies with DHH users, and by aiming for keeping the interface as generic as possible not to restrict application scenarios. Since the accuracy of the recognition performance greatly influences the experience, the system should show reasonable accuracy without requiring users to manually record a large number of audio clips. In addition, besides providing a recording functionality using a microphone, the system should also allow users to incorporate training samples from external resources to support a diversity of sound classes.

In the training phase, the user first defines a new target class (Fig. 3(a)). To add training data to the target class, the user can either record sound clips with a microphone (Fig. 3(b)) or select training samples from the AudioSet dataset [11] (Fig. 3(c)). AudioSet is a large-scale dataset of 10-seconds sound clips extracted 


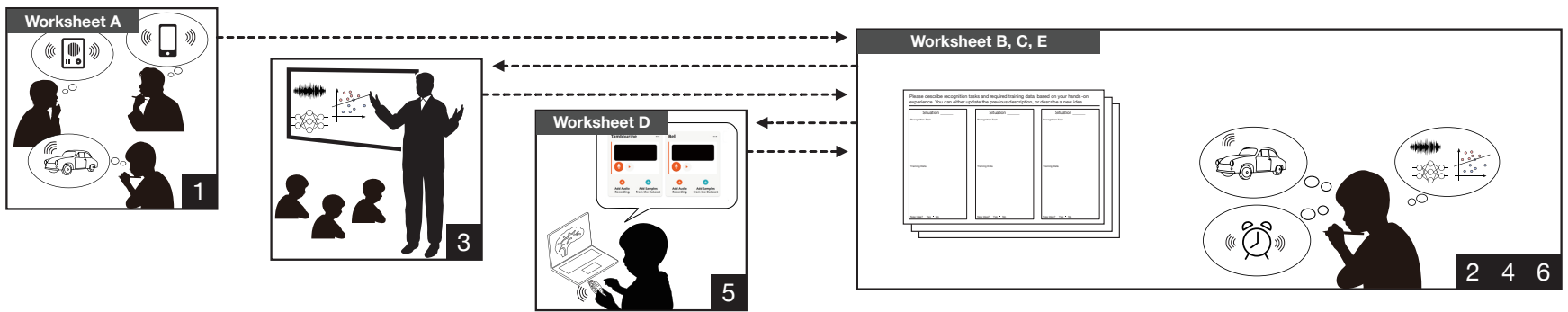

Figure 2: Our workshop consists of six phases. The main parts are the phases where the participants describe their ideas concerning sound recognition tasks (phases 2,4 , and 6 ), and these parts are repeated after a discussion among the participants about their daily-life demands (phase 1), a short lecture on ML (phase 3), and hands-on experience with our interactive learning system (phase 5).

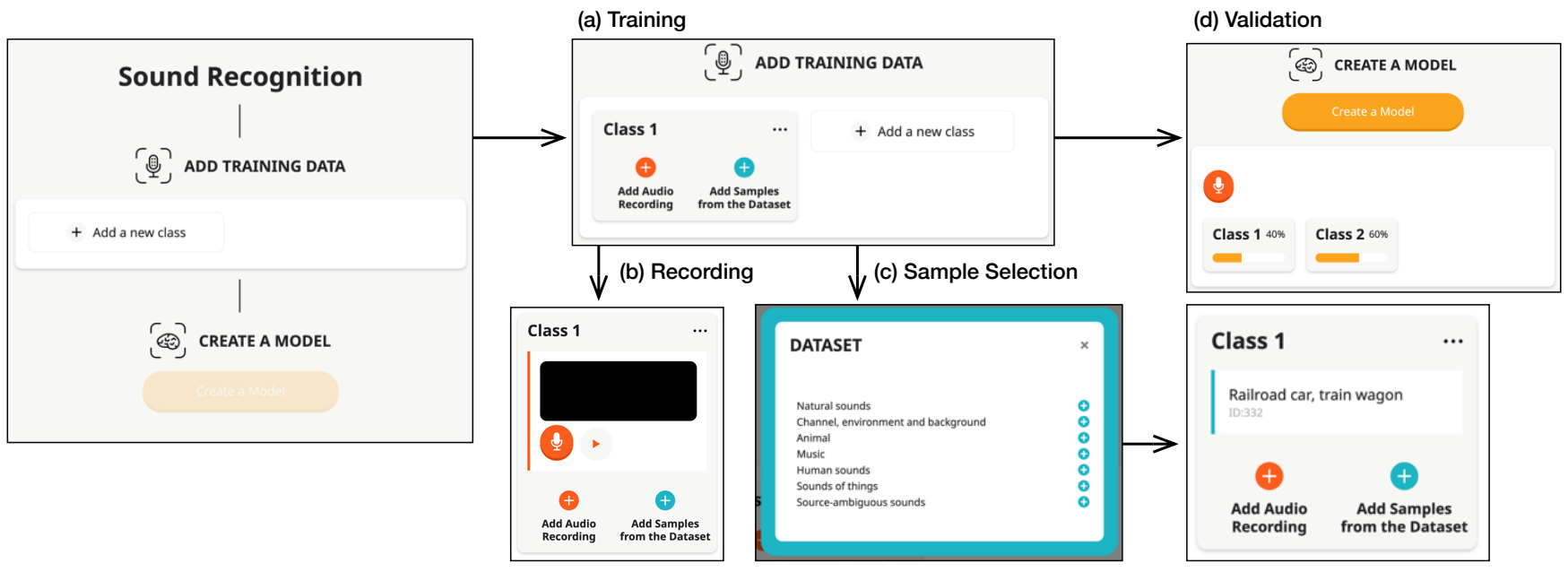

Figure 3: GUI and flow of the interactive learning system. In the training phase, (a) the user first defines a new class and then adds training audio samples by either (b) recording live audio with an internal microphone or (c) selecting samples from the AudioSet dataset. After model training, (d) the system shows real-time classification results with class probabilities, and the user can validate the system performance.

from YouTube videos and consists of 2.1 million sound clips with human-annotated labels of 632 audio event classes. The use of this dataset extends the capability of the interactive learning system and provides users with a practical understanding of a typical ML workflow using existing datasets. When selecting samples from the dataset, a user can navigate through the AudioSet ontology and press the plus button to add the desired class. If the user selects an intermediate class on the ontology tree, all subclasses are also added to the user-defined class. Then, the user triggers the model training button, and the training data are sent to the ML backend. After model training, the system shows real-time classification results with class probabilities (Fig. 3(d)) and the user can then validate the system performance.

The backend is implemented using the scikit-learn [29] and TensorFlow libraries [1]. We use the VGGish deep neural network pre-trained on AudioSet for feature extraction [14]. The network takes a mel spectrogram of $16-\mathrm{kHz}$ resampled audio as input. The recorded samples are processed with a 0.96-s sliding window, and each sample corresponds to 64 mel bands and 96 frames of 10 ms each. The embedded features from the network are further post-processed by applying a PCA transformation [17] and 8-bit quantization, which results in a 128-dimensional feature vector for every $0.96 \mathrm{~s}$. In other words, if a user records an $N$-second sound clip using the internal microphone, it is split into approximately $N$ segments and each segment is used as a training sample. For the samples from AudioSet, we used the pre-computed 128-dimensional feature vectors. Using the deep features, the system trains the sound recognition model using the Random Forest algorithm [5]. In our implementation, the number of trees is set to 30 . The Gini impurity is used as a metric, with a maximum depth of 10 and a minimum number of samples per leaf node of 1 .

\subsection{Lecture and Demonstration}

In the fourth phase, a short undergraduate-level short lecture is given by a lecturer. The goal of the lecture is not only to provide 
an overview but also to explain the limitations of ML approaches. The lecture takes approximately 30 minutes, and the presentation is given in the native language of participants (Japanese) with automatic subtitling and sign-language interpretation. Three interpreters take part throughout our workshop, and interpreted the content spoken by the presenters in turn. While the interpreters are not ML experts, the slides used in the lecture are shared with them prior to the lecture. Presentation handouts are also distributed to the participants.

The lecture is prepared to cover the following topics: 1) a brief overview of media recognition and artificial intelligence, 2) an introduction to ML, 3) the difficulties and limitations of ML, and 4) the basics of deep learning. The basic concept of artificial intelligence is explained using image and sound recognition as example tasks, and a simple linear classification between coffee and milk is explained step by step to illustrate the basic ML process. This example of coffee/milk classification is further used to explain the difficulties of ML by discussing, e.g., the ambiguity of latte and the differences in the required training data for various drink classification tasks. For completeness, how deep learning differs from traditional ML approaches is further explained.

After the lecture, we provide a hands-on demonstration of the interactive learning system to explain its basic usage. This is done by demonstrating the training process for several different sound recognition tasks. We first train a classifier discriminating between toy bells and human voices using live audio recordings. At this point, we explicitly explain that environmental noise is also classified into either the bell or voice class in this simple, two-class setting, and demonstrate the importance of introducing a third class corresponding to environmental noise. To demonstrate the usage of the AudioSet training data, we also train a human voice category classifier by selecting samples from the dataset.

\subsection{Participants}

Table 1 lists the details of the workshop participants. We recruited twelve DHH people (5 female) through social network services and individual e-mails. While the participants did not include any ML experts, there were five participants who had experiences of programming. This is because we primarily contacted DHH user groups on social network services, and most of the candidates had a certain level of interest in technology. The participants were compensated for about $\$ 90$ for the time they spent in the workshop (9 hours including interview), which was decided based on the minimum wage in Tokyo, Japan (workshop venue) and their travel costs. They were, on average, 38.3 years old $(\mathrm{SD}=15.7$, ranging 18 64). Five had profound hearing loss and another five had severe hearing loss. Eight participants reported congenital hearing loss. Most of the participants used hearing aid devices: nine used digital hearing aids and two reported cochlear implants. Three (P7, P9, and P10) also participated in a prototype preliminary study to implement the interactive system mentioned in subsection 3.2.

\section{DATA AND ANALYSIS}

In this section, we summarize the data collected during the workshop, and describe the details of the data analysis.

\subsection{Data Collection}

After the workshop, we conduct semi-structured interviews to obtain details on the described contents and the changes in the participants' subjective views during the workshops. The interview is designed to last 30 minutes but ranged from 25 to 34 minutes. We use text chatting when vocal communication is difficult. After being shown the worksheets, the participants are asked for the detailed meanings of their descriptions. For the worksheets describing recognition task ideas (Worksheets $\mathrm{B}, \mathrm{C}$, and $\mathrm{E}$ ), the participants are also asked whether they notice technical difficulties or contradictions in their descriptions. All interviews are recorded with a video and/or a text log.

Before the workshop, we ask the participants to fill out an online survey concerning their disability levels and experience with computer programming. After the workshop, we use another online survey to collect data concerning their impressions of the workshop, the lecture, and the interactive system. The questions described in Section 3 are asked in these online surveys. We record audiovisual data of the participants' behavior during the workshop, and collect the system logs of the interactive learning system.

\subsection{Coding}

The data recorded in the semi-structured interviews are transcribed and coded by one of the authors. Then the same author iteratively develops a codebook through discussions with the another authors to identify detailed patterns and similarities. In addition, to understand the effects of the lecture and use of the interactive system, two authors independently code the results from Worksheets B, C, and $\mathrm{E}$ and iteratively developed a codebook. Worksheet $\mathrm{D}$ is coded and analyzed together with the video recorded during phase 5 and the system logs. The codebook for the semi-structured interviews consists of 15 codes, while that for Worksheet D involves four viewpoints: 1) the difference between the detection and classification tasks, 2) the existence of negative classes for detection tasks, 3) the existence of unnecessary class definitions, and 4) the necessity of diverse training data.

\section{RESULTS AND FINDINGS}

In this section, we describe findings from the coding results of Worksheets B-E, and semi-structured interviews. The findings are summarized from mainly four perspectives: ML understanding, expressed task categories, task formulations, and effects of collaboration.

\subsection{Technical Understanding of ML}

We first describe how participants accept ML technology from the perspective of their understanding of training data.

5.1.1 Recognizing the Necessity of Diverse Data. Table 2 shows the summary of worksheet coding. We describe the coding results for participants with and without programming experiences separately in Table 2. It can be seen that, in general, participants gradually perceived the concept of ML-based on the need for the training data. All participants mentioned the need for diverse data to train the ML system after the usage of the interactive system, while at first only half of the participants mentioned it. 
Table 1: Details of the workshop participants.

\begin{tabular}{cccccc}
\hline ID & Gender & Age & Disability Level & Hearing Aid Devices & Programming \\
\hline P1 & F & 20 & Moderate (congenital) & Hearing aid & None \\
P2 & F & 51 & Profound (congenital) & Nothing & None \\
P3 & M & 50 & Severe (congenital) & Hearing aid & $10 \mathrm{Y}$ \\
P4 & F & 43 & Moderate (post-lingual) & Hearing aid & None \\
P5 & M & 40 & Profound (congenital) & Hearing aid & $3 \mathrm{Y}$ \\
P6 & F & 60 & Severe (post-lingual) & Wireless microphone & None \\
P7 & M & 18 & Profound (congenital) & Cochlear implant & $1 \mathrm{Y}$ \\
P8 & M & 64 & Severe (congenital) & Hearing aid & None \\
P9 & M & 21 & Profound (congenital) & Hearing aid & $3 \mathrm{Y}$ \\
P10 & M & 32 & Severe (congenital) & Hearing aid & None \\
P11 & F & 45 & Profound (post-lingual) & Cochlear implant & None \\
P12 & M & 27 & Severe (post-lingual) & Hearing aid & $2 \mathrm{M}$
\end{tabular}

Table 2: Summary of worksheet coding. The fourth column shows the number of participants who set non-ML tasks, and the fifth and sixth columns show the cumulative number of participants who mentioned the need for diverse data and data collection.

\begin{tabular}{clcc|cc}
\hline Programming Experience & Worksheet ID & Total Num. & Non-ML Tasks & Need for Diverse Data & Data Collection \\
\hline \multirow{2}{*}{ With } & B (Second phase) & 5 & $0(0.0 \%)$ & $4(80.0 \%)$ & $5(0.0 \%)$ \\
& C (Fourth phase) & 5 & $0(0.0 \%)$ & $5(100 \%)$ & $1(20.0 \%)$ \\
& E (Sixth phase) & 5 & $0(0.0 \%)$ & $5(100 \%)$ & $2(28.6 \%)$ \\
\hline \multirow{2}{*}{ Without } & B (Second phase) & 7 & $3(42.9 \%)$ & $4(57.1 \%)$ & $4(0.0 \%)$ \\
& C (Fourth phase) & 7 & $1(14.3 \%)$ & $6(85.7 \%)$ & $5(71.4 \%)$
\end{tabular}

For participants without programming experience, this tendency was especially remarkable. From the beginning to the end of the workshop, the percentage of the number of participants without programming experience who mentioned the need increased 71.4 percentage points ( 5 people out of 7 ) while that of participants with programming experience increased only 20 percentage points $(1$ person out of 5). For example, P6, a hard-of-hearing participant without programming experience described a task as "AI that recognizes various electric sounds and lets me know the sounds" at the beginning on Worksheet B. On Worksheet $\mathrm{E}$ after using the interactive system, she mentioned the potential improvement in the accuracy with diverse data as "by training the system with as diverse data as possible, the accuracy of the system will be improved." Conversely, while the total number of participants who mentioned diverse data increased for participants with programming experience, the tendency was not very clear. The need for diverse data was recognized by four participants $(\mathrm{P} 5, \mathrm{P} 7, \mathrm{P} 9$, and $\mathrm{P} 12)$ even before the ML lecture, and the other participant (P3) recognized this right after the lecture.

5.1.2 Imagining the Training Data Collection Process. Through the workshop, participants also started to consider data collection approaches. As can be seen in Table 2, the total number of participants who commented on data collection increases during the workshop, and this effect is more significant among the participants without programming experience. While no one mentioned data collection approaches at the beginning, five participants (P2, P4, P6, P10, and P11) commented on data collection after using the interactive system. After the lecture, P6 added details concerning the training data collection as the need to "search for the high pitched sound of a phone call on the Internet and train the system" on Worksheet C.

The participants also pointed out some positive effects of interacting with the system to recognize the importance of training data collection. It encouraged them to think over what they learned in the lecture, made them recognize the importance of recording training data under diverse environments, and helped them imagine the existence of noise in environmental sounds. For example, P12, who is the only participant with programming experience mentioning the taring data collection, said that "While using the system (, I recognized the necessity of mixing environmental sounds into the training data). I thought that it would be useless if I recorded only the sounds of moving cars (, which were the sound to be detected)." P10 pointed out the benefit of using such an interactive, personal sound recognition system in daily life: “...At the beginning, it would probably not be very accurate, but it would improve as people became familiar (with the system). So, [...] it is better (for people) to train the system by themselves from scratch." 
5.1.3 Facing Difficulties of Data Collection. Three participants (P1, P3, P5) further recognized the difficulties in collecting diverse data. Related to diverse data, in the semi-structured interview after the workshop, one participant (P1, without programming experience) had some negative opinions on the necessity of diverse data when using the system in her daily life. She mentioned that she realized that the system requires a lot of training data and that it would be very time-consuming to record data with the interface.

The participants also have difficulties in collecting data as they want using the system mainly due to the limitation in the GUI design. As described before, the participants could use training samples from the AudioSet dataset to train the recognition system. However, the AudioSet data was used only by six participants (P2, $\mathrm{P} 5$, and $\mathrm{P} 9-\mathrm{P} 12)$. While there are some positive reactions to the dataset ("(The AudioSet data) were easy to understand. (This is because) the dataset was divided, into male and female voices, and so on."(P9)), some participants reported that they could not find the audio samples that they needed.

Additionally, nine participants (P1-P7, P10, and P12) searched for relevant videos on YouTube and recorded audio samples by playing videos on their own smartphones. During the interview, P7 suggested that it would be useful if there was a functionality to register sounds from YouTube ("I think that it is better if I can register sound data directly from YouTube."). P5 reported that he checked the ontology tree but did not use the AudioSet data because he had difficulties in searching through it. P11 pointed out that the GUI resulted in information overload, saying that "There are too many (categories), and I couldn't decide which one I wanted." P12 checked its actual audio samples and mentioned the quality of the dataset. He said that "... When I checked the original data on YouTube, there were many irrelevant sounds."

Another difficulty in collecting data is because of participants' disabilities, and three participants (P2, P4, and P6) reported difficulties in checking the training sounds. Even though this poses a chicken-and-egg problem for sound recognition, P6 mentioned the necessity of visualization or semantic information: "Without transforming (sounds) to color or graphs that I can see or vibrations, I don't have any means of recognizing them, so I can't check the recorded sounds. It would probably be preferable to display the waveform. The best thing would be to display texts showing that my cell phone is ringing now."

\subsection{Differences and Changes in Expressed Tasks}

Next, we describe the tasks obtained from the worksheet coding in detail. By analyzing differences among workshop phases, we clarify the participants' sound perception and how it changed through the workshop.

5.2.1 Differences in Task Categories. In Fig. 4, we classified userexpressed recognition tasks into 16 categories except for the tasks which are not recognized as ML tasks. Non-ML tasks include, for example, a system to transfer signals to a cashier at a cafe that does not need any semantic recognition. We divided the participants into two groups: deaf people (people with profound disabilities) and hard-of-hearing people (people with severe or moderate disabilities).
Figure 4 shows the fractions of participants in each group describing the task categories.

As can be seen in Fig. 4, hard-of-hearing people expressed more task categories (14 categories) than deaf people (11 categories). As well as the number of expressed categories, there are also differences in expressed contents between deaf and hard-of-hearing people in the task descriptions. For example, only deaf people expressed the needs for Sounds of nature and Environmental sounds, such as the sounds of raining/wind and environmental noise in crowded places. On the other hand, there are five categories which were described by only hard-of-hearing people. For example, deaf participants did not describe tasks corresponding to Abnormal sounds such as noises made by the crowd in a dangerous situation. This indicates the tendency that deaf people have difficulties in describing tasks that can be perceived mainly as acoustic phenomena, and differences in the sound experience between deaf and hard-of-hearing people can influence the variations in the task description.

5.2.2 Changes in the Number of Expressed Tasks. In Fig. 5, we analyzed the change of the number of expressed task categories by each group of participants. Although the decrease and increase of the number of expressed categories do not directly indicate positive or negative effects, we use this metric to understand how participants changed their thoughts in the course of the workshop. The participants are divided into groups based on the difference in disability levels (deaf or hard-of-hearing) and programming experiences (with or without programming experience).

In Fig. 5, we can observe a tendency that the participants first came up with diverse tasks, but later they discarded some and focused on refining parts of the ideas. As the results of the entire participants, the number of expressed categories increases after the lecture (Worksheet C) and decreases after the interactive learning (Worksheet E). With deaf people, the number of expressed task categories tends to decrease through the workshop regardless of the programming experience. On the other hand, the number of the categories expressed by hard-of-hearing people increases after the lecture of ML, while it decreases (or stay the same for those with programming experience) after the interactive learning.

\subsection{Differences in Task Formulations}

Next, we summarize further details of the expressed tasks on worksheet $\mathrm{D}$ (written before and during phase 5) by focusing on the differences in the task formulation during the use of the interactive system.

5.3.1 Classification and Detection. As shown in Table 3, five participants (P1, P2, P7, P8, P12) described classification tasks to categorize input sounds into different categories, and eight participants (P1, P3-6, P9, P10, P12) described detection tasks to find target sound events from the input audio stream. Practically speaking, classification systems assumes that the user already knows that a particular sound event is happening, and s/he asks the system which category the input sound belongs to. On the other hand, detection systems have only two categories: positive or negative, and the system tells the user whether the current sound is corresponding to the target (positive) category or not. 


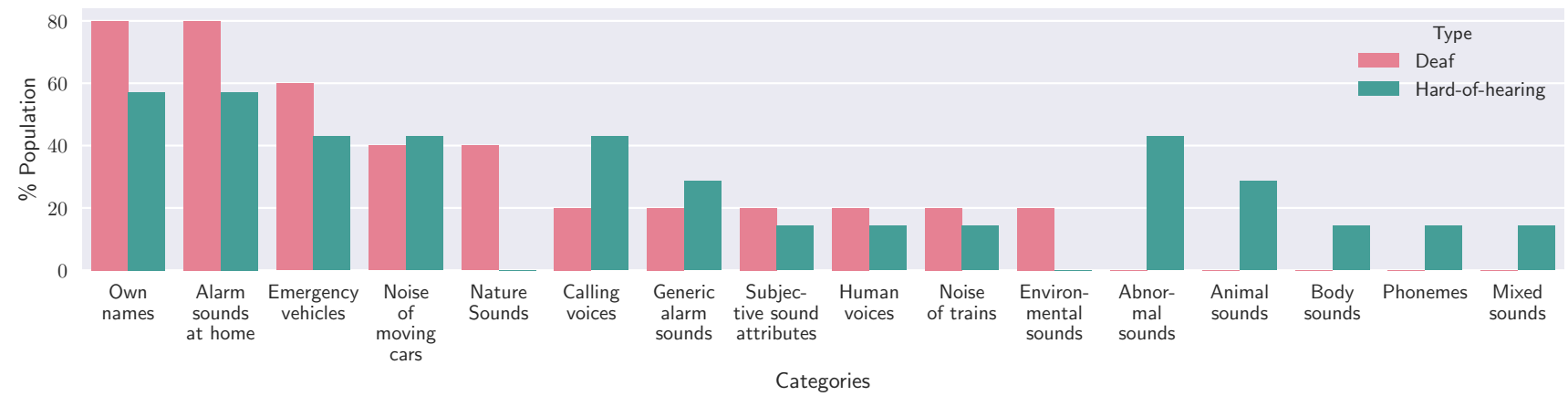

Figure 4: Expressed ML task categories on three worksheets (Worksheet B, C, and E). Each plot shows the fraction of deaf and hard-of-hearing participants who described each task category. This does not include the ones we categorized as non-ML tasks.

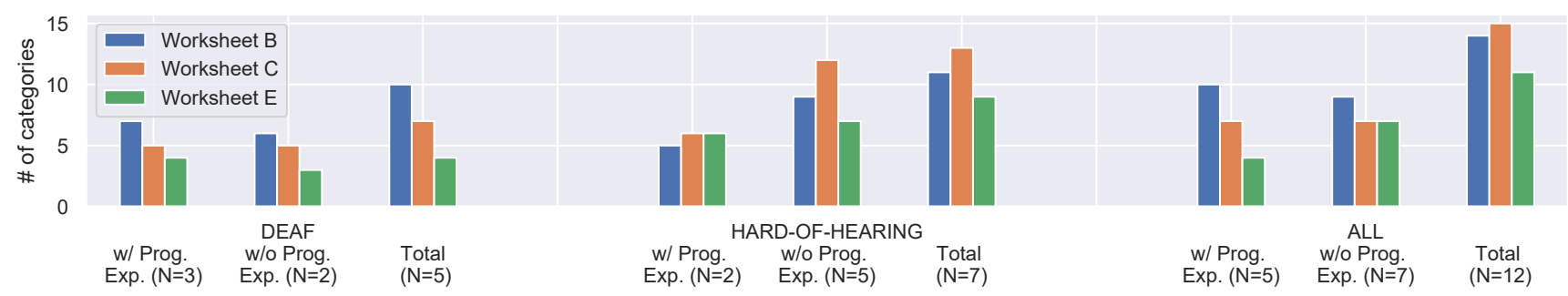

Figure 5: The number of tasks expressed in each worksheet by each group of participants. Participants are categorized based on the existence of programming experience (with or without programming experiences), and disability levels (deaf or hardof-hearing). The numbers of participants in each category are shown in the labels.

Table 3: The number of classification and detection tasks expressed by each group in Worksheet $D$ while using the interactive system. $\mathrm{N}$ indicates the number of participants categorized into each group (each participant can express multiple tasks).

\begin{tabular}{|c|c|c|c|c|c|c|c|c|c|}
\hline Disability & \multicolumn{3}{|c|}{ Deaf } & \multicolumn{3}{|c|}{$\mathrm{HoH}$} & \multicolumn{3}{|c|}{ Total } \\
\hline Programming Experience & $\mathrm{N}$ & Classification & Detection & $\mathrm{N}$ & Classification & Detection & $\mathrm{N}$ & Classification & Detection \\
\hline With & 3 & $1(33.3 \%)$ & $2(66.7 \%)$ & 2 & $0(0.0 \%)$ & $2(100 \%)$ & 5 & $1(20.0 \%)$ & $4(80.0 \%)$ \\
\hline Without & 2 & $2(100 \%)$ & $0(0.0 \%)$ & 5 & $2(40.0 \%)$ & $4(80.0 \%)$ & 7 & $4(57.1 \%)$ & $4(57.1 \%)$ \\
\hline Total & 5 & $3(60.0 \%)$ & $2(40.0 \%)$ & 7 & $2(28.6 \%)$ & $6(85.7 \%)$ & & & \\
\hline
\end{tabular}

The participants with programming experience or hard-of-hearing participants tend to describe detection tasks. 6 participants of hardof-hearing and 4 with programming experience described detection tasks. Although four participants (P1, P4, P5, P12) failed to assign negative classes, we confirmed through the interviews that their original intentions were detection. For example, the intended task for P13 was vehicle detection on the road, however he set multiple classes corresponding to different vehicle types without any negative classes. On the other hand, some participants successfully introduced negative classes if it corresponded to a concrete sound category. For example, P1, who tried to detect male vocal solos from musical songs successfully introduced classes corresponding to female/chorus/instrumental parts.
5.3.2 Difficulty of Defining Class Categories. We also found that the participants often introduced overly subdivided classes into recognition tasks. Five participants (P1, P3, P5, P9, P12) set more than two labels for the detection tasks. For example, P3 wanted a recognition system to detect voices calling his name and introduced four classes: voices calling his name in 1) quiet places, 2) crowded places, and 3) a hospital and 4) environmental noise in an office. While P3 successfully introduced a negative class for this detection task, he split the positive class (i.e., voices calling his name) into three different classes for different environments. In the semi-structured interview, he told us that he thought that the recognition accuracy would improve by subdividing the classes and he did not want to set an additional environment classification task. 
These two observations indicate that the main difficulty is defining abstract sound categories.

\subsection{Collaborations during Workshop}

In our workshop, the participants had opportunities to collaborate with other participants when describing their daily problems and using interactive systems. In this subsection, we summarize the effect of such collaborations.

5.4.1 Realizing Possibilities of Sound Usage. We found that some participants (P1, P4, P10) started to think about new ways of using sounds to train ML models based on collaboration with other participants. When participants expressed their daily problems in phase 1 , one participant (P10) mentioned that he overcame his difficulties in expressing their daily problems with the collaboration with another participant. P10 commented that "At first, nothing came to my mind at all. [...] There are certainly some things that were inconvenient, but I've never felt really troubled by them." And he said “...But when I exchanged my sheet with the person next to me, something like 'electrical sounds (are difficult to hear)' was written, and I noticed that I actually didn't think (of this as a problem)." This implies that participants could realize such problems through collaboration with other participants with different disabilities and life experiences.

Collaboration with other participants with respect to the use of the interactive systems also influences how they recognized sounds. One participant $(\mathrm{P} 4)$ reported that she recognized that the potential of sound recognition was more than what she had expected: "Because my period of being away from sounds was long, my ideas about what could be done with sound had not grown. [...] Two other people (who trained the model with ML in the same room) believed in the possibilities of sound and tried to do several things by making various sounds." She almost forgot what is possible with recognizing sounds, but she realized the importance of sound to recognize surrounding environments by collaborating with other participants. This implies that our workshop approach had an effect on the perception of sound itself, and made participants recognize the possibilities of sound recognition.

5.4.2 Learning How to Use the Interactive System. While using the interactive system, participants obtained ideas concerning how to use and train the system effectively. In our workshop, participants were allowed to communicate with other participants as they wanted. As the simplest effect of collaboration, some participants (e.g., P4) started to use YouTube videos to train the model after finding other participants used YouTube videos as the source of sounds.

These collaborations also had the effect of letting other participants know of effective ways of training the system. One participant (P3), who has a 10-year programming experience asked other participants to call his name to create various sounds of his name. After such a collaborative work to train his system, P1, who did not have any programming experiences, defined a category corresponding to noisy sounds as the negative class to her target class on Worksheet E. She expressed the reason for setting the noise class as "I came up with the idea of setting the class of noise because) I made noise with another person who tried to make the noise class." This means that, if there was no collaboration with other participants, she would have never found the effective way of training the system. While in phase 3 (ML lecture), the necessity of negative class was explicitly mentioned by giving some examples and handouts, through collaboration and communication with other participants, the whole level of understandings about the concept of ML improved. This indicates the effect of collaboration between people who have various levels of experience in information technology.

\section{DISCUSSION}

In this section, we discuss how the knowledge gap on ML and sounds can be bridged with the workshop. We also summarize important aspects of designing the workshop and system, together with difficulties and future challenges.

\subsection{Bridging the Knowledge Gap}

During the workshop, we observed that the knowledge gap due to the disability and the lack of technological understandings were gradually bridged. In terms of technological understandings, it can be seen with the fact that there were no participants describing non-ML tasks in worksheet E. Our findings that non-expert people perceive ML technology from the perspective of the necessity of diversity of data supports Yang et al. [39] that mentioned the tendency of non-expert people to add more data to improve ML model performance. In terms of disability and sound perception, some participants commented that they came to know how to use the sounds to train their recognition systems. Some also mentioned that, from the collaborations with other participants and the use of the interactive system, they learned what kind of sound information can be useful in their daily lives. DHH people always live in environments where there are no or only a small amount of sounds, their daily-life behaviors are often adapted to such circumstances and they can manage to do many things they want to do. Although The absence of some sound categories has not been recognized as "daily-life problems" by themselves, through the collaboration with other participants who experience different sound environments, they realize some new ideas to improve their daily lives.

From our analysis of the workshop, there are possibilities that the understanding of ML affects the perception of sounds and its potential usage. For the hard-of-hearing participants, the number of expressed task categories increased after the ML lecture and the interactive learning. On the other hand, the number of task categories expressed by deaf people continued to decrease after both the lecture and the usage of the system. And the total numbers of expressed task categories are different between deaf (11 categories) and hard-of-hearing participants (14 categories). This difference also supports a prior study [4]. Bragg et al. point out that there were differences between deaf and hard-of-hearing people in the requirements for sounds, depending on the prior experience in the auditory environment in the way that there are meaningful differences in the characteristics of expressed recognition tasks. Our findings imply that the ML lecture facilitated hard-of-hearing participants to imagine new ideas of using sound. However, once they have a more practical understanding of ML by using the interactive system, they might also learn the difficulties in training the system, and the number of their expressed tasks decreases. 
Our findings also implicate that there are two types of task formulations while using interactive systems; classification and detection. Participants with programming experience tend to describe detection tasks (4 people out of 5). This is possibly because it is easier for people with prior knowledge about information technology to recognize their intending tasks are detection. At the same time, hard-of-hearing participants tend to describe detection tasks (6 people out of 7). A possible explanation is that hard-of-hearing people can perceive the existence of sound to some extent and thus they could be more interested in identifying whether the current sound is corresponding to the category of interest.

\subsection{Workshop Design}

We found that the task descriptions and the acceptance of ML by participants differed depending on their disability levels and prior knowledge on information technology. Nonetheless, most of the participants grasped the importance of diverse training data by using the system. By interacting with the ML system, they started to imagine use-case scenarios in more realistic ways and to recognize the importance of designing training data acquisition processes.

In terms of efficiencies in the use of interactive systems and recognition task expression, collaboration with other $\mathrm{DHH}$ people can greatly improve the outcome. One of the most important aspects of our workshop design was that it provided an opportunity for participants to learn, discuss, and collaborate with people having different levels of disabilities and experiences in information technology. As we pointed out in Section 5, collaboration with other participants in the phases of task expression and interactive learning helped them recognize the existence of various sounds and their related recognition tasks. Moreover, while using the interactive system, participants collaboratively found effective ways to use the system, such as the use of YouTube videos to record training samples. Physical collaboration with other participants to record real sounds also had a positive effect on the improvement in task expressions. These findings, conversely, indicate the limitation of simply providing such an interactive system to individual users alone. Both workshop settings and interactive systems should be designed for a group of people who collaborate with each other.

\subsection{Design of the Interactive System}

Our findings indicate that participants accepted the concept of ML mainly from the perspective of training data collection and of what kind of data should be used to train. This is understandable considering that, in practice, what has to be done by users is establishing their training dataset when users personalize their sound recognition systems. Participants could have perceived the process of training data collection by using the system, and they gradually recognized the necessity of diverse data to train a robust recognition model.

The participants' recognition of ML also implicates that it is important to design the GUI for interactive ML so that users can easily check the quality of audio samples as training data. The importance is also endorsed by the requirements for the GUI to support participants' disabilities. In our workshop, participants could have checked the quality of data only by watching the source YouTube videos. However, there are often mismatches in audio and video contents on YouTube and this is a critical issue, especially for deaf participants. Additionally, when participants physically made sounds for themselves (by using, e.g., bells), some participants reported that it was very difficult to confirm the existence of a sound, i.e., whether a target event actually makes a sound. Even though this poses a fundamental challenge in this scenario, one potential factor that influenced the user experience was user feedback. While our system did not provide any feedback about recorded or imported sounds, it could potentially help users grasp the audio content by, e.g., displaying spectrogram. During the interactive machinelearning process, the intermediate visualization of sound clips is also important, in addition to a visualization of the recognition results.

\subsection{Challenges}

Finally, we summarize some shortcomings and essential challenges to our approach. One of the most essential challenges is that, regardless of whether the person has prior knowledge on information technology or ML, it is still difficult for $\mathrm{DHH}$ people to come up with task definitions to recognize sounds that they cannot perceive. For example, one participant (P8) was able to describe a task to recognize music categories because he enjoyed music before he lost his hearing. However, this task could not be described by participants with congenital profound hearing loss. Both technological developments and community support will be required to address this issue.

Second, our GUI was designed to represent classification tasks and could not express the full potential of sound recognition and ML. Although the participants could define various recognition and detection tasks, it is almost impossible to extend the task formulation to, e.g., regression tasks. While we adopted classification because it can be seen as the most representative ML formulation, this can certainly restrict the imagination of participants and there were few regression-like ideas in the task descriptions. An important future work is to explore interactions between users and systems with interfaces designed intuitively without over-simplifying the diverse formulations of ML.

Finally, we were not able to assist non-expert participants enough to express more complex scenarios which were difficult to be described as detailed ML tasks. To develop assistive systems from diverse use-case scenario descriptions by $\mathrm{DHH}$ people, it is also important to break down more abstract scenario descriptions into concrete sensing tasks. While there are cases where ML is not required at all, some scenarios have good potential to investigate creative applications of ML. As ML is becoming a commodity even for novice users in recent years, there will be more opportunities for $\mathrm{DHH}$ people to learn and experience ML technologies and more creative ideas will come through. Our study also indicates that user-friendly interactive ML systems can serve as a good starting point for non-expert users.

\section{CONCLUSIONS}

In this study, we analyzed the changes in the perception of sound and ML by non-expert DHH people through a workshop with lecture and interactive learning sessions. From the analyses of expressed tasks, we found that participants accept the concept of ML 
from the perspective of training data. We also clarified that the level of disabilities and the experience in information technology (more specifically, programming experience) influence the understanding of ML. Collaborations among participants with diverse levels of disabilities and experiences on information technology resulted in more efficient technical understanding. We found that participants recognize the effective use of sounds to train ML systems and solve their daily problems by collaborating with other participants and interacting with the system. This study illustrates both the potential and challenges of democratizing ML technologies in the context of assistive technologies.

\section{ACKNOWLEDGMENTS}

This work was supported by JST CREST Grant Number JPMJCR1781, Japan.

\section{REFERENCES}

[1] Martín Abadi, Paul Barham, Jianmin Chen, Zhifeng Chen, Andy Davis, Jeffrey Dean, Matthieu Devin, Sanjay Ghemawat, Geoffrey Irving, Michael Isard, Manjunath Kudlur, Josh Levenberg, Rajat Monga, Sherry Moore, Derek G. Murray, Benoit Steiner, Paul Tucker, Vijay Vasudevan, Pete Warden, Martin Wicke, Yuan Yu, and Xiaoqiang Zheng. 2016. TensorFlow: A System for Large-scale Machine Learning. In Proceedings of the 12th USENIX Conference on Operating Systems Design and Implementation (Savannah, GA, USA) (OSDI'16). 265-283.

[2] Saleema Amerashi, Maya Cakmak, William Bradley Knox, and Todd Kulesza. 2014. Power to the People: The Role of Humans in Interactive Machine Learning. AI MAGAZINE 535, 4 (2014). https://doi.org/10.1609/aimag.v35i4.2513

[3] Saleema Amershi, James Fogarty, Ashish Kapoor, and Desney Tan. 2009. Overview Based Example Selection in End User Interactive Concept Learning. In Proceedings of the 22Nd Annual ACM Symposium on User Interface Software and Technology (Victoria, BC, Canada) (UIST '09). 247-256. https://doi.org/10.1145/1622176. 1622222

[4] Danielle Bragg, Nicholas Huynh, and Richard E. Ladner. 2016. A Personalizable Mobile Sound Detector App Design for Deaf and Hard-of-Hearing Users. In Proceedings of the 18th International ACM SIGACCESS Conference on Computers and Accessibility (Reno, Nevada, USA) (ASSETS '16). ACM, New York, NY, USA, 3-13. https://doi.org/10.1145/2982142.2982171

[5] Leo Breiman. 2001. Random Forestss. Machine Learning 45, 5 (2001), 6-32. https://doi.org/10.1023/A:1010933404324

[6] Sachin Chachada and C.-C. Jay Kuo. 2013. Environmental sound recognition: A survey. In 2013 Asia-Pacific Signal and Information Processing Association Annual Summit and Conference (Kaohsiung, Taiwan). https://doi.org/10.1109/APSIPA. 2013.6694338

[7] Mari Carmen Domingo. 2012. An overview of the Internet of Things for people with disabilities. Journal of Network and Computer Applications 35, 2 (2012), $584-$ 596. https://doi.org/10.1016/j.jnca.2011.10.015 Simulation and Testbeds.

[8] B. M. Eskofier, S. I. Lee, J. Daneault, F. N. Golabchi, G. Ferreira-Carvalho, G. Vergara-Diaz, S. Sapienza, G. Costante, J. Klucken, T. Kautz, and P. Bonato. 2016. Recent machine learning advancements in sensor-based mobility analysis: Deep learning for Parkinson's disease assessment. In 2016 38th Annual International Conference of the IEEE Engineering in Medicine and Biology Society (EMBC). 655658. https://doi.org/10.1109/EMBC.2016.7590787

[9] Jerry Alan Fails and Dan R. Olsen Jr. 2003. Interactive machine learning. In Proceedings of the 8th international conference on Intelligent user interfaces (Miami, Florida, USA) (IUI '03). 39-45. https://doi.org/10.1145/604045.604056

[10] Rebecca Fiebrink, Perry R. Cook, and Dan Trueman. 2011. Human Model Evaluation in Interactive Supervised Learning. In Proceedings of the SIGCHI Conference on Human Factors in Computing Systems (Vancouver, BC, Canada) (CHI '11) 147-156. https://doi.org/10.1145/1978942.1978965

[11] Jort F. Gemmeke, Daniel P.W. Ellis, Dylan Freedman, Aren Jansen, Wade Lawrence, R. Channing Moore, Manoj Plakal, and Marvin Ritter. 2017. Audio Set: An ontology and human-labeled dataset for audio events. In 2017 IEEE International Conference on Acoustics, Speech and Signal Processing (ICASSP). https://doi.org/ 10.1109/ICASSP.2017.7952261

[12] Claudio Guida, Dario Comanducci, and Carlo Colombo. 2011. Automatic Bus Line Number Localization and Recognition on Mobile Phones-A Computer Vision Aid for the Visually Impaired. In Image Analysis and Processing - ICIAP 2011, Giuseppe Maino and Gian Luca Foresti (Eds.). Springer Berlin Heidelberg, Berlin, Heidelberg, 323-332.
[13] Kotaro Hara, Christine Chan, and Jon E. Froehlich. 2016. The Design of Assistive Location-based Technologies for People with Ambulatory Disabilities: A Formative Study. In Proceedings of the 2016 CHI Conference on Human Factors in Computing Systems (CHI '16). 1757-1768. https://doi.org/10.1145/2858036.2858315

[14] Shawn Hershey, Sourish Chaudhuri, Daniel P. W. Ellis, Jort F. Gemmeke, Aren Jansen, Moore R. Channing, Manoj Plakal, Devin Platt, Rif A. Saurous, Bryan Seybold, Malcolm Slaney, Ron J. Weiss, and Kevin Wilson. 2017. CNN Architectures for Large-Scale Audio Classification. In 2017 IEEE International Conference on Acoustics, Speech and Signal Processing (ICASSP) (New Orleans, LA, USA). https://doi.org/10.1109/ICASSP.2017.7952132

[15] Gahangir Hossain and Mohammed Yeasin. 2012. Cognitive Load Based Adaptive Assistive Technology Design for Reconfigured Mobile Android Phone. In Mobile Computing, Applications, and Services, Joy Ying Zhang, Jarek Wilkiewicz, and Ani Nahapetian (Eds.). Springer Berlin Heidelberg, Berlin, Heidelberg, 374-380.

[16] Dhruv Jain, Angela Lin, Rose Guttman, Marcus Amalachandran, Aileen Zeng, Leah Findlater, and Jon Froehlich. 2019. Exploring Sound Awareness in the Home for People Who Are Deaf or Hard of Hearing. In Proceedings of the 2019 CHI Conference on Human Factors in Computing Systems (Glasgow, Scotland Uk) (CHI '19). Association for Computing Machinery, New York, NY, USA, Article 94, 13 pages. https://doi.org/10.1145/3290605.3300324

[17] Ian Jolliffe. 2011. Principal Component Analysis. 1094-1096. https://doi.org/10. 1007/978-3-642-04898-2 455

[18] Hernisa Kacorri. 2017. Teachable Machines for Accessibility. SIGACCESS Access. Comput. 119 (Nov. 2017), 10-18. https://doi.org/10.1145/3167902.3167904

[19] Hernisa Kacorri, Kris M. Kitani, Jeffrey P. Bigham, and Chieko Asakawa. 2017. People with Visual Impairment Training Personal Object Recognizers: Feasibility and Challenges. In Proceedings of the 2017 CHI Conference on Human Factors in Computing Systems (Denver, Colorado, USA) (CHI '17). 5839-5849. https: //doi.org/10.1145/3025453.3025899

[20] Shaun K. Kane, Amy Hurst, Erin Buehler, Patrick A. Carrington, and Michele A. Williams. 2014. Collaboratively Designing Assistive Technology. Interactions 21, 2 (March 2014), 78-81. https://doi.org/10.1145/2566462

[21] Simon Katan, Mick Grierson, and Rebecca Fiebrink. 2015. Using Interactive Machine Learning to Support Interface Development Through Workshops with Disabled People. In Proceedings of the 33rd Annual ACM Conference on Human Factors in Computing Systems (Seoul, Republic of Korea) (CHI '15). 251-254. https://doi.org/10.1145/2702123.2702474

[22] Saba Kawas, George Karalis, Tzu Wen, and Richard E. Ladner. 2016. Improving Real-Time Captioning Experiences for Deaf and Hard of Hearing Students. In Proceedings of the 18th International ACM SIGACCESS Conference on Computers and Accessibility (Reno, Nevada, USA) (ASSETS '16). Association for Computing Machinery, New York, NY, USA, 15-23. https://doi.org/10.1145/2982142.2982164

[23] Todd Kulesza, Margaret Burnett, Weng-Keen Wong, and Simone Stumpf. 2015. Principles of Explanatory Debugging to Personalize Interactive Machine Learning. In Proceedings of the 20th International Conference on Intelligent User Interfaces (Atlanta, Georgia, USA) (IUI '15). Association for Computing Machinery, New York, NY, USA, 126-137. https://doi.org/10.1145/2678025.2701399

[24] Todd Kulesza, Simone Stumpf, Margaret M. Burnett, and Irwin Kwan. 2012. Tell me more?: the effects of mental model soundness on personalizing an intelligent agent. In Proceedings of the SIGCHI Conference on Human Factors in Computing Systems (Austin, Texas, USA) (CHI '12). 1-10. https://doi.org/10.1145/2207676. 2207678

[25] Todd Kulesza, Weng-Keen Wong, Simone Stumpf, Stephen Perona, Rachel White, Margaret M. Burnett, Ian Oberst, and Andrew J. Ko. 2009. Fixing the program my computer learned: barriers for end users, challenges for the machine. In Proceedings of the 14th international conference on Intelligent user interfaces (Sanibel Island, Florida, USA) (IUI '09). 187-196. https://doi.org/10.1145/1502650.1502678

[26] Nicholas D. Lane, Petko Georgiev, and Lorena Oendro. 2015. DeepEar: Robust Smartphone Audio Sensing in Unconstrained Acoustic Environments Using Deep Learning. In Proceedings of the 2015 ACM International foint Conference on Pervasive and Ubiquitous Computing (Osaka, Japan) (UbiComp '15). Association for Computing Machinery, New York, NY, USA, 283-294. https://doi.org/10. $1145 / 2750858.2804262$

[27] F. J. M. Meiland, B. J. J Hattink, T. Overmars-Marx, M. E. de Boer, A. Jedlitschka, P. W. G. Ebben, Ir. I. N. W. Stalpers-Croeze, S. Flick, J. van der Leeuw, I. P. Karkowski, and R. M. Dröes. 2014. Participation of end users in the design of assistive technology for people with mild to severe cognitive problems; the European Rosetta project. International Psychogeriatrics 26, 5 (May 2014), 769-779. https://doi.org/10.1017/S1041610214000088

[28] Kayur Patel, James Fogarty, James A. Landay, and Beverly Harrison. 2008. Investigating Statistical Machine Learning as a Tool for Software Development. In Proceedings of the SIGCHI Conference on Human Factors in Computing Systems (Florence, Italy) (CHI '08). Association for Computing Machinery, New York, NY, USA, 667-676. https://doi.org/10.1145/1357054.1357160

[29] Fabian Pedregosa, Gaël Varoquaux, Alexandre Gramfort, Vincent Michel, Bertrand Thirion, Olivier Grisel, Mathieu Blondel, Peter Prettenhofer, Ron Weiss, Vincent Dubourg, Jake Vanderplas, Alexandre Passos, David Cournapeau, Matthieu Brucher, Matthieu Perrot, and Édouard Duchesnay. 2011. Scikit-learn: Machine Learning in Python. J. Mach. Learn. Res. 12 (Nov. 2011), 2825-2830. 
[30] Yi-Hao Peng, Ming-Wei Hsi, Paul Taele, Ting-Yu Lin, Po-En Lai, Leon Hsu, Tzu-chuan Chen, Te-Yen Wu, Yu-An Chen, Hsien-Hui Tang, and et al. 2018. SpeechBubbles: Enhancing Captioning Experiences for Deaf and Hard-of-Hearing People in Group Conversations. In Proceedings of the 2018 CHI Conference on Human Factors in Computing Systems (Montreal QC, Canada) (CHI '18). Association for Computing Machinery, New York, NY, USA, Article 293, 10 pages. https://doi.org/10.1145/3173574.3173867

[31] M. Philipose, K. P. Fishkin, M. Perkowitz, Patterson D. J., D. Fox, H. Kautz, and D. Hahnel. 2004. Inferring activities from interactions with objects. IEEE Pervasive Computing 3, 4 (October-December 2004), 50-57. https://doi.org/10.1109/MPRV 2004.7

[32] Martha E. Pollack. 2005. Intelligent Technology for an Aging Population: The Use of AI to Assist Elders with Cognitive Impairment. AI MAGAZINE 26, 2 (2005), 9-24. https://doi.org/10.1609/aimag.v26i2.1810

[33] Liu Sicong, Zhou Zimu, Du Junzhao, Shangguan Longfei, Jun Han, and Xin Wang. 2017. UbiEar: Bringing Location-independent Sound Awareness to the Hard-of-hearing People with Smartphones. Proceedings of the ACM on Interactive Mobile, Wearable and Ubiquitous Technologies 1, 2, Article 17 (June 2017), 24 pages. https://doi.org/10.1145/3090082

[34] P. Strumillo. 2010. Electronic interfaces aiding the visually impaired in environmental access, mobility and navigation. In 3rd International Conference on Human System Interaction. 17-24. https://doi.org/10.1109/HSI.2010.5514595

[35] Justin Talbot, Bongshin Lee, Ashish Kapoor, and Desney S. Tan. 2009. EnsembleMatrix: Interactive Visualization to Support Machine Learning with
Multiple Classifiers. In Proceedings of the SIGCHI Conference on Human Factors in Computing Systems (Boston, MA, USA) (CHI '09). 1283-1292. https: //doi.org/10.1145/1518701.1518895

[36] Joe Tullio, Anind K. Dey, Jason Chalecki, and James Fogarty. 2007. How It Works: A Field Study of Non-Technical Users Interacting with an Intelligent System. In Proceedings of the SIGCHI Conference on Human Factors in Computing Systems (San Jose, California, USA) (CHI '07). Association for Computing Machinery, New York, NY, USA, 31-40. https://doi.org/10.1145/1240624.1240630

[37] F. Vidal-Verdu and M. Hafez. 2007. Graphical Tactile Displays for VisuallyImpaired People. IEEE Transactions on Neural Systems and Rehabilitation Engineering 15, 1 (March 2007), 119-130. https://doi.org/10.1109/TNSRE.2007.891375

[38] W. Xiong, L. Wu, F. Alleva, J. Droppo, X. Huang, and A. Stolcke. 2018. The Microsoft 2017 Conversational Speech Recognition System. In 2018 IEEE International Conference on Acoustics, Speech and Signal Processing (ICASSP). 5934-5938. https://doi.org/10.1109/ICASSP.2018.8461870

[39] Qian Yang, Jina Suh, Nan-Chen Chen, and Gonzalo Ramos. 2018. Grounding Interactive Machine Learning Tool Design in How Non-Experts Actually Build Models. In Proceedings of the 2018 Designing Interactive Systems Conference (Hong Kong, China) (DIS '18). Association for Computing Machinery, New York, NY, USA, 573-584. https://doi.org/10.1145/3196709.3196729

[40] Chien Wen Yuan, Benjamin V. Hanrahan, Sooyeon Lee, Mary Beth Rosson, and John M. Carroll. 2017. Constructing a holistic view of shopping with people with visual impairment: a participatory design approach. Universal Access in the Information Society (October 2017), 1-14. https://doi.org/10.1007/S10209-0170577-1 